\title{
La enseñanza de las matemáticas, un reto para los maestros del siglo $\mathrm{XXI}^{1}$
}

\author{
Guillermo López-Quijano² \\ Universidad Pedagógica y Tecnológica de Colombia, Tunja, Colombia \\ loz.qui@hotmail.com
}

1 Artículo de investigación derivado del proceso de reflexión e investigación desarrollado durante dos años al interior del curso de Maestría en Educación, en temas de ambiente de aprendizaje colaborativo, el sistema didáctico aprendizaje basado en problemas, las características del aula especializada y el proceso de evaluación formativa.

2 Especialista en Docencia de la Matemática; Candidato a Magister en Educación, Universidad Pedagógica y Tecnológica de Colombia. 


\title{
La enseñanza de las matemáticas, un reto para los maestros del siglo XXI
}

\section{Resumen}

El presente artículo trata, en una primera parte, sobre la problemática a la que se enfrentan docentes, estudiantes y padres de familia cuando emprenden el proceso de enseñanza y aprendizaje de las matemáticas, y de las dificultades que el discente tiene a lo largo de su vida estudiantil para aplicar el conocimiento matemático en la solución de situaciones problema de su entorno; en una segunda parte se presenta una propuesta didácticopedagógica, que tiene como objetivo evaluar la actitud socio-académica del estudiante frente al aprendizaje de las matemáticas en los alumnos de la Institución Educativa Técnica Rafael Uribe, del municipio de Toca. La propuesta envuelve cuatro aspectos: el ambiente de aprendizaje colaborativo, el sistema didáctico aprendizaje basado en problemas, las características del aula especializada y el proceso de evaluación formativa. Por último, se presentan los resultados obtenidos a través del tipo de investigación sistematización de experiencias educativas, desde el punto de vista actitudinal, emocional y socio-académico del estudiante.

Palabras clave: Ambiente de aprendizaje colaborativo, aprendizaje basado en problemas, aula especializada, evaluación formativa.

\section{The teaching of mathematics, a challenge for teachers of XXI century}

\begin{abstract}
This article is, in the first part, about the problems that teachers, students and parents face when they embark on the process of teaching and learning of mathematics, and about the difficulties that the learner encounters throughout his or her educational life in which he or she applies mathematical knowledge in solving environmental problems. The second part presents a didactics and pedagogical proposal which aims at assessing the social-academic attitude of the student during the learning of mathematics among students of Rafael Uribe Technical School from Toca municipality. The proposal involves four aspects: the collaborative learning environment, the didactic system based on problem-solving, the characteristics of the specialized classroom and the formative assessment. Lastly, the results obtained from this type of research, systematization of Teaching experiences, from an attitudinal, emotional, and social - academic aspect of the student.
\end{abstract}

Key words: Collaborative Learning Environment; learning based on problems; specialized classroom, formative assessment.

\section{O ensino das matemáticas, um desafio para os professores do século XXI.}

\section{Resumo}

O presente artigo estuda, em uma primeira parte, o problema que enfrentam os docentes, estudantes e padres de família no momento em que empreendem o processo de ensino e aprendizagem das matemáticas, e as dificuldades que o aluno tem ao longo da sua vida estudantil para aplicar o conhecimento matemático na solução de situações difíceis do seu entorno; em uma segunda parte, apresenta-se uma proposta didático pedagógica, que tem como objetivo avaliar a atitude sócio-acadêmica do estudante frente à aprendizagem das matemáticas nos alunos da Instituição Educativa Técnica Rafael Uribe, do município de Toca. A proposta contém quatro aspectos: o ambiente de aprendizagem colaborativa, o sistema didático aprendizagem baseada em problemas, as características da aula especializada e o processo de avaliação formativa. Finalmente, apresentam-se os resultados obtidos por meio do tipo de pesquisa sistematização de experiências educativas, desde a perspectiva emocional, sócio acadêmico e da atitude do estudante.

Palavras chave: ambiente de aprendizagem colaborativa; aprendizagem baseada em problemas; aula especializada; avaliação formativa 


\section{Introducción}

La educación actual exige que en el marco del Proyecto Educativo Institucional (PEI) se implementen ambientes y entornos pedagógicos viables, que permitan a los estudiantes ver claramente la articulación del saber construido en el aula con el quehacer cotidiano en su entorno sociocultural, familiar y, a corto plazo, en el campo laboral, para coadyuvar así al desarrollo propio y de la comunidad (MEN, 1994).

Tradicionalmente se enseñan contenidos y temas que se considera que todos los niños y niñas deben conocer, la revolución educativa plantea que lo importante no es solo conocer, sino también saber y saber hacer, es decir, aplicar lo que se sabe para desempeñarse en una situación de la vida real. Por su parte, las matemáticas, debido a su carácter abstracto, necesitan de ambientes propios, que estimulen en el estudiante el desarrollo del pensamiento matemático y faciliten el proceso de enseñanza y aprendizaje, además, las matemáticas requieren de estrategias didáctico-pedagógicas que despierten la curiosidad, el interés y el gusto por la materia, y que desvirtúen el temor que estas producen en los estudiantes (Fandiño, 2006). En el mismo sentido, se requiere que el saber matemático esté relacionado constantemente con la praxis cotidiana desde tempranos años escolares, para que el alumno, durante su desarrollo mental, la asimile y la haga parte de él, como herramienta que facilite numerosos procesos en la interacción con el medio.

Las matemáticas hacen parte del propósito de formación de los estudiantes, y se plasman en el currículo de las instituciones educativas, su enseñanza y aprendizaje se inicia en los primeros años escolares, y se extiende hasta la universidad, de modo que se convierte en una asignatura fundamental y obligatoria, especialmente en la educación básica y media (MEN, 1994). A partir del estudio de la matemática, los estudiantes desarrollan procesos mentales que les permitan reconstruir, construir, afianzar y aplicar este conocimiento en el momento que el medio lo requiera.

Además, existen causas que hacen de la enseñanza y aprendizaje de las matemáticas un proceso tedioso y poco significativo, como por ejemplo: cuando no existen en el aula los medios para este fin; los espacios para la interacción entre docente y estudiantes, a lo largo del desarrollo de las temáticas, no son los adecuados; los recursos didácticos no son pertinentes o no se cuenta con ellos en el momento oportuno; las pedagogías y metodologías con las que se desarrolla la clase no están en consonancia con los objetivos trazados para la misma; los aportes de la evaluación al aprendizaje de las matemáticas son escasos o nulos, entre otras. Entonces, el articular de forma reflexiva este conjunto de elementos de la educación, pueden llegar a convertir el aula de clase en un verdadero recinto donde se construya conocimiento de una forma dinámica, libre y en continua relación 
con el medio. De esta forma, el aprendizaje de las matemáticas pueda convertirse en eje fundamental, que potencie habilidades, capacidades y competencias en los estudiantes, de modo que reconozcan su importancia a partir de la utilidad práctica (D’Amore et ál., 2008), y logren una trascendencia del conocimiento aprendido y construido en el aula más allá de los muros institucionales.

\section{Problemática}

Por diversas razones, la enseñanza y aprendizaje de las matemáticas se ha convertido en un verdadero reto para los maestros y estudiantes, e indirectamente para los padres de familia, desde los primeros años de escolaridad, al respecto, D'Amore et ál. (2008) dice: "lo que aleja a los estudiantes de la matemática no es ella misma en sí, sino la forma como esta se les presenta, la falta de interacción entre el mundo real y los contenidos orientados en el aula; ellos se desestimulan cuando descubren que la matemática que se enseña en la escuela no se relaciona con la vida cotidiana", de modo que se produce un bloqueo en el desarrollo de su vida escolar.

De la misma forma, la didáctica de las matemáticas ha demostrado que estas son accesibles, y aun agradables, si su enseñanza se hace mediante una adecuada orientación, que implique una permanente interacción entre el maestro y sus alumnos, y entre estos y el entorno. Entonces, que a través de la exploración, abstracción, clasificación, medición y estimación, entre otros, sean capaces de llegar a resultados que permitan comunicarse en forma matemática y descubrir que estas se encuentran íntimamente relacionadas con la realidad y con las situaciones que los rodean (MEN, 2009).

Lo anterior invita a los educadores a reflexionar sobre las prácticas en el aula y a proponer ambientes didáctico-pedagógicos acordes con el entorno sociocultural del estudiante, de tal forma que tanto él como el docente puedan construir o reconstruir conocimiento coherente con las condiciones de un mundo cada vez más exigente, cambiante y globalizado. En este sentido, surge la pregunta: ¿cómo se afecta la actitud del estudiante de básica secundaria y media frente al aprendizaje de las matemáticas, al incluir en las prácticas educativas elementos didáctico-pedagógicos que generen ambientes de clase diferentes a los tradicionales?

\section{Propuesta didáctico-pedagógica}

En la propuesta, que he denominado "entorno para el aprendizaje de las matemáticas", articulo cuatro elementos: un espacio acondicionado con las características de un aula especializada; el sistema didáctico del aprendizaje basado en problemas (ABP); la estrategia de estudio grupal del ambiente de aprendizaje colaborativo, y la evaluación para el aprendizaje como mecanismo de retroalimentación en la construcción de saberes. 
La propuesta se desarrolla en la Institución Educativa Técnica Rafael Uribe, del municipio de Toca, Boyacá, con alumnos de grado séptimo (47 niños), de décimo (49 jóvenes) y de undécimo (56 jóvenes), cuyo objetivo central fue evaluar la actitud socioacadémica y actitudinal del estudiante frente al aprendizaje de las matemáticas, al desempeñarse en un ambiente de aula distinto al tradicional.

El enfoque cualitativo de la investigación ha sido base fundamental en el desarrollo del proyecto, puesto que permitió una continua interacción entre estudiante, docente y entorno, al desarrollar actividades en las que involucra diferentes ambientes socioeducativos. El tipo de investigación, bajo el cual se llevó a cabo el estudio de la aplicación de la propuesta, es la sistematización de ejercicios educativos, puesto que permite materializar las experiencias en su capacidad de reordenar, reorientar y cualificar la acción educativa. Esta involucra actores de la educación, saberes, teorías, contextos y prácticas que se desarrollan bajo una lógica en el círculo de la experiencia, para la consecución de respuestas a interrogantes que se generan a lo largo del proceso investigativo (Mejía, 2011).

Para la recolección de la información se han utilizado diversas técnicas: como el diario de campo, donde se registra detalladamente lo ocurrido en cada una de las clases; la observación directa, videograbaciones, en las que se registran actitudes de los estudiantes frente a la metodología aplicada en las clases; fotografías; encuestas de opinión; entrevistas individuales; resultados de talleres y actividades realizadas por los estudiantes. Para el manejo de la información se han empleado las técnicas de procesamiento y análisis de datos cualitativos (Mejía, 2011).

\section{Espacio acondicionado en el entorno para el aprendizaje de las matemáticas}

Los propietarios de almacenes de toda índole con frecuencia destinan recursos económicos para marketing, publicidad y mejoramiento del entorno físico de su negocio, para captar la atención de las personas que por allí transitan, con el fin de aumentar las ventas; de igual forma, cuando se desea celebrar el cumpleaños de un ser querido, la casa se decora para la ocasión con los elementos apropiados, si es hombre o mujer y dependiendo de su edad; se sabe que la decoración le da esplendor, personalidad y más alegría a la fiesta, y no es difícil percibir la actitud de felicidad del festejado en ocasiones como estas. Al llevar estas sencillas pero eficaces acciones al aula de clase, de inmediato se nota el cambio de expresión en el rostro de los estudiantes y en su forma de actuar: ¡es la sala de matemáticas! "Qué chévere", dicen algunos, otros solo sonríen, otros observan con sumo cuidado los elementos que se encuentran en el lugar, pero hay algo en común: ¡todos quieren estar en ese lugar! 
Para la experiencia se ha seleccionado un salón amplio, 60 metros cuadrados aproximadamente, con buena iluminación y aireado. Una de las paredes de menor superficie se adecuó, parte de ella como pantalla de proyecciones, y otra parte para ubicar un tablero acrílico, en el cual el estudiante refuerza sus conocimientos, además, se fijaron frases que invitan a la superación personal e intelectual a todos las personas que visiten el aula. Las tres paredes restantes son para fijar y exponer los trabajos y proyectos que clase a clase los estudiantes elaboran, y que además sirven como material didáctico para otros cursos o para recordar conocimientos que por no ser frecuentados se van olvidando. Este material es expuesto hasta que el espacio del salón se agote, y los estudiantes necesiten publicar lo elaborado sobre otra temática.

Otro aspecto importante en la adecuación del aula, consiste en dotarla de mesas y sillas cómodas, de forma que el estudiante pueda ubicarse en el lugar que más le agrade, con sus compañeros de más confianza (no más de cuatro) o con quienes se siente apoyado a lo largo del desarrollo de las clases. También se organizó en el salón un biblio-banco, con textos de matemáticas de todos los grados, ya que el estudiante necesita consultar temas nuevos, recordar conocimientos olvidados, aclarar dudas o simplemente ojear los textos que le parezcan llamativos en el momento que lo desee, y qué mejor que sea dentro de la misma clase, orientado y apoyado por el docente. También se tienen a la mano, otros recursos didácticos, como planchas prediseñadas, escuadras, compás, bloques matemáticos, dinero didáctico, entre otros, que resultan bastante útiles y oportunos para dar explicación a eventos inesperados o no planeados de la clase.

La conexión a internet, el manejo del computador y el video beam en las clases se han constituido en un apoyo significativo, a través de ellos se pueden estructurar actividades que saquen a docente y estudiantes de la rutina, y mejorar de esta manera el ambiente y la atención a la clase, al igual la comunicación entre estudiantedocente y estudiante-estudiante.

\section{El aprendizaje basado en problemas como estrategia pedagógica en el entorno para el aprendizaje de las matemáticas}

Uno de los grandes retos que se ha propuesto la educación, en todos los tiempos, es el de favorecer la construcción de conocimiento y facilitar el aprendizaje, hazaña que requiere de pedagogías que permitan a los estudiantes, desde su contexto, desarrollar competencias y potenciar habilidades para adaptarse a una sociedad en continua transformación. Al igual que sucede en otras ciencias, en el campo de la matemática se han propuesto diversos enfoques pedagógicos y métodos que invitan a reflexionar sobre el proceso de enseñanza y aprendizaje, con esto se busca que los estudiantes se interesen cada vez más por construir su propio conocimiento. 
El aprendizaje basado en problemas (ABP) se ha adoptado como elemento pedagógico central, que brinda al docente que se desempeña en el entorno para el aprendizaje de las matemáticas estrategias didáctico-pedagógicas pertinentes, para que el estudiante logre construir su propio conocimiento. Escribano \& Del Valle (2010) definen el ABP como un "sistema didáctico que requiere que los estudiantes se involucren de forma activa en su propio aprendizaje, hasta el punto de definir un escenario de formación auto-dirigida", donde el elemento central en el proceso de enseñanza y aprendizaje es el estudiante, quien usa problemas como base fundamental para construir e integrar el conocimiento. Barrows \& Tamblyn (1980) establecen algunas características del método, enfatizan en que el aprendizaje es auto-dirigido, centrado en el alumno y se produce en pequeños grupos, los profesores son facilitadores del proceso, y los problemas son el centro para el estímulo del aprendizaje y vehículo para el desarrollo de habilidades para resolverlos.

Este método sugiere un aprendizaje autorregulado e integrado en el sentido de que relaciona el qué con el cómo y el para qué se aprende. Hay que dejar que el estudiante explore por sí mismo las posibilidades para solucionar los problemas, y es en este proceso donde construye o reconstruye el conocimiento, en una acción comunicativa de él con el grupo y con el profesor (Escribano \& Del Valle, 2010).

Asimismo, Hmelo (2004) define el ABP como un "sistema curricular e instruccional que desarrolla simultáneamente tanto la estrategia propia de resolución de un problema como las bases del conocimiento y habilidades específicas propias de una disciplina", en donde el estudiante aprende en forma activa con los demás integrantes del grupo y colabora en la búsqueda de la respuesta al problema planteado. Hmelo (2004) recalca que este sistema curricular está concebido para que el alumno desarrolle habilidades para analizar los problemas de manera metódica, y desempeñar con éxito las distintas funciones de los miembros del grupo, como ser tutor, coordinador y secretario, además, el método permite que el alumno lleve a cabo las actividades individuales complementarias de una forma segura y creativa.

Para desarrollar con éxito el método ABP y que el estudiante logre un aprendizaje significativo, Escribano \& Del Valle (2010) proponen un proceso que han denominado "los siete pasos", donde inicialmente se hace la presentación del problema, luego se aclaran los términos que el estudiante no conozca, en seguida se identifican los elementos constituyentes del problema, después se establecen posibles soluciones a través de hipótesis, se continúa con la identificación de vacíos en el conocimiento, para luego acceder a la información necesaria que lleve a la resolución del problema o a la identificación de nuevos problemas, y se finaliza así el proceso o se repite el ciclo de nuevo. El papel del estudiante, durante este proceso de aprendizaje auto-dirigido o autorregulado, es el de enfrentarse en un comienzo a un problema supervisado por un tutor, y analizar el problema individualmente o en forma grupal. Este análisis lleva a preguntas sobre las que el estudiante construye el conocimiento 
o logra el aprendizaje a través de la consulta o preguntando al profesor, por último, los estudiantes informan sobre lo que han aprendido y evalúan sus avances en la resolución del problema origen del proceso.

En el mismo sentido, el papel del profesor en el marco del ABP, según Escribano \& Del Valle (2010), es el de ser gestor del proceso, el que diseña instructivos claros, comprensibles e interdisciplinares. Él debe pensar la materia desde quien ha de aprenderla, sobre todo saber cómo aprenden sus estudiantes, prever desde dónde se puede abordar mejor el problema, con qué tipo de dificultades pueden encontrarse a lo largo del proceso, qué tipo de apoyo o ayudas pueden necesitar los alumnos para que progresen en el aprendizaje, en consecuencia, el profesor aparece como una pieza fundamental en la dinamización del proceso de aprendizaje y en el fomento de un ambiente de trabajo colaborativo.

\section{Ambientes colaborativos en el entorno para el aprendizaje de las matemáticas}

Dillenbourg (1999) plantea que la noción de colaborar para aprender en la educación escolar tiene significado amplio, en el que se contempla la posibilidad de trabajar en una situación educativa en forma contraria al aprendizaje individual, en el cual aparecen las interacciones entre los estudiantes en el desarrollo de las actividades propuestas en clase, y es de gran importancia la estructura del diálogo colaborativo. Fernández \& Melero (1995) señalan que en el trabajo colaborativo se hace una distribución más equitativa del conocimiento entre el educador y los estudiantes, en la que se espera que las responsabilidades sean compartidas. Así se invita a los estudiantes a definir los objetivos específicos dentro de la temática que se está enseñando, y se brindan opciones para realizar actividades que logren atraer su atención y los animen a evaluar lo que han aprendido, es decir, que el aprendizaje colaborativo busca el trabajo en pequeños grupos, con el fin de que los estudiantes interactúen con los compañeros para maximizar su propio aprendizaje y el de los demás (Johnson et ál., 1993).

De acuerdo con Collazos \& Mendoza (2006), el aprendizaje colaborativo es, ante todo, un sistema de interacciones cuidadosamente diseñado, que organiza e induce la influencia recíproca entre los integrantes de un equipo. En el contexto educativo es un modelo de aprendizaje interactivo, en el que se invita a caminar juntos, a sumar esfuerzos y una serie de estrategias que permiten alcanzar los objetivos propuestos, ya que el énfasis de este aprendizaje está en el pensamiento crítico, la resolución de problemas y la construcción del conocimiento (Bruffee, 1995).

Collazos \& Mendoza (2006) resaltan que la colaboración podrá ser efectiva si hay una interdependencia genuina entre los estudiantes que están colaborando, 
en la que se describe como: La necesidad de compartir información que lleve a entender conceptos y obtener conclusiones; la necesidad de dividir el trabajo en papeles complementarios, y finalmente la necesidad de compartir el conocimiento en términos explícitos.

\section{La evaluación en el "entorno para el aprendizaje de las matemáticas"}

Expresiones como "saquen una hojita", "mañana hay evaluación de todo lo visto hasta la fecha", "la próxima semana se inician los exámenes bimestrales", entre otras, se escuchan en las instituciones educativas con bastante frecuencia; las actividades académicas se suspenden, para dar paso a la evaluación de lo aprendido por los estudiantes durante determinado tiempo escolar; estén o no preparados, todos reciben pliego el día del examen. El estudiante, confiando en su buena suerte, marca la equis o llena el óvalo donde cree que es correcto, entrega, y el docente califica muy rápido, pues se sabe de memoria las respuestas. Al poco tiempo, cada estudiante sabe su nota, y los pliegos van a la basura, en forma de avioncito o pelotica, después de esto se inicia un nuevo tema o simplemente se reanudan las clases, para continuar por un camino la enseñanza y por otro distinto la evaluación, como dos extrañas.

De esta manera, el estudiante es el único responsable de los resultados: si son malos, es él quien debe cambiar, lo demás, currículo y prácticas educativas, continúa inamovible, luego la evaluación se convierte en una obligación que afecta solo al estudiante (Santos, 1995).

Este esquema evaluativo puede ser funcional para examinar determinados procesos de instrucción, pero la pregunta sería: ¿esta forma de evaluar contribuye a la construcción del conocimiento, a potenciar habilidades, desarrollar competencias en el estudiante y a la formación integral de la persona? Santos (1995), al referirse a la naturaleza de la evaluación, afirma que esta debe ser procesual y no meramente final, es decir, se debe realizar durante el proceso académico y no solo cuando se termine el programa, debe ser una evaluación al servicio del estudiante, que lo ayude en el proceso de aprendizaje y formación personal.

Asimismo, para Santaibáñez (2001), la evaluación es un procedimiento intencionado, funcional, sistemático, continuo e integral, con el fin de obtener información tendiente a mejorar o aumentar la eficacia de los procedimientos educativos: es funcional, ya que se realiza con base en objetivos; sistemático, porque está inmerso en un sistema global, del cual hace parte la comunidad educativa; continuo, porque se realiza a lo largo de todo el proceso educativo y no al final de cada periodo escolar, e integral, debido a que se considera al estudiante dentro 
de un contexto educativo, donde se evalúan sus conocimientos y comportamientos de tipo afectivo y psicomotor; luego la evaluación debe usarse no para eliminar estudiantes, sino para guiar su proceso de aprendizaje (Santaibáñez, 2001).

En el mismo sentido, Gardner (1995), en su artículo La evaluación en su contexto, afirma que la alternativa a los test estandarizados enfatiza en la forma y el momento oportuno para practicar la evaluación: "en lugar de imponerla de forma externa en momentos inoportunos durante el curso, la evaluación tendría que formar parte del entorno natural del aprendizaje, debería tener lugar como parte del interés del individuo en una situación de aprendizaje"; de esta forma, la evaluación se convierte en un proceso continuo en el aula, para encaminar al estudiante hacia un mejor aprendizaje de manera voluntaria.

En este sentido, la evaluación que se plantea en el entorno para aprendizaje de las matemáticas, donde se conjugan los sistemas didáctico-pedagógicos, aula especializada, aprendizaje basado en problemas y ambiente para el aprendizaje colaborativo, es de carácter formativo, la cual está presente en el proceso de construcción del conocimiento en el estudiante (Puentes, 2005). Este tipo de evaluación hace parte de las prácticas pedagógicas cotidianas en el aula, y una de sus finalidades es la de retroalimentar tanto al docente como al estudiante, para dar continuamente la oportunidad de corregir los errores cometidos en los procesos de enseñanza y aprendizaje (Santaibáñez, 2001). Otra finalidad de este tipo de evaluación es la de obtener información acerca de las competencias y habilidades de los alumnos, con el objetivo de proporcionar datos y respuestas útiles a los estudiantes evaluados y a la comunidad que los rodea (Gardner, 1995). En consecuencia, la evaluación se constituye en un proceso que evalúa para el aprendizaje, es decir, no es una valoración de resultados, sino una evaluación que contribuye al proceso educativo, hace parte inherente de este.

En el entorno para el aprendizaje de las matemáticas, la evaluación indica el nivel de comprensión y desempeño del estudiante en cada uno de los temas que componen el plan de estudio de la asignatura, lo mismo que las deficiencias cognitivas y errores cometidos por él en el proceso educativo. De igual forma, la evaluación representa para el profesor un indicativo, que lo invita a reflexionar sobre sus prácticas educativas, en busca del mejoramiento continuo en el aprendizaje de sus alumnos, es decir, "se trata, en último término, de mirar la evaluación como otro evento de aprendizaje" (Torres, 1996).

Por otra parte, la metodología de aprendizaje basado en problemas, desarrollada en un ambiente para el aprendizaje colaborativo, admite un proceso de evaluación continuo, a través de una serie de pasos de retroalimentación constante, en el que la técnica de estudio grupal se convierte en el epicentro del aprendizaje, y en donde se fortalecen las competencias y habilidades del estudiante. 
El proceso de evaluación comienza en el mismo momento en que se inician las actividades de la clase, el docente explica la ruta que se debe seguir durante la misma, y las metas que el estudiante debe alcanzar en cada una de sus etapas. Durante las actividades propuestas para desarrollar en el aula, el profesor acompaña de cerca a cada grupo, momento en el que él recomienda bibliografía, sugiere procedimientos, formula preguntas de control de procesos, motiva al grupo a seguir o controla la disciplina, en caso de ser necesario. Estas acciones permiten que haya un diálogo constructivo a lo largo de la clase, donde el estudiante manifiesta sus saberes, dificultades e inquietudes frente al aprendizaje, de una forma directa o indirecta, a través de las expresiones orales, escritas o por medio de las gesticulaciones. Estos momentos son aprovechados por el docente para corregir errores, afianzar procedimientos y fortalecer la confianza en sí mismo de cada estudiante y del grupo en general: El diálogo con el alumno en los grupos de trabajo sirve para identificar dónde se encuentran las deficiencias en el aprendizaje y elaborar actividades diferentes para reconducir la enseñanza (López y Hinojosa, 2001).

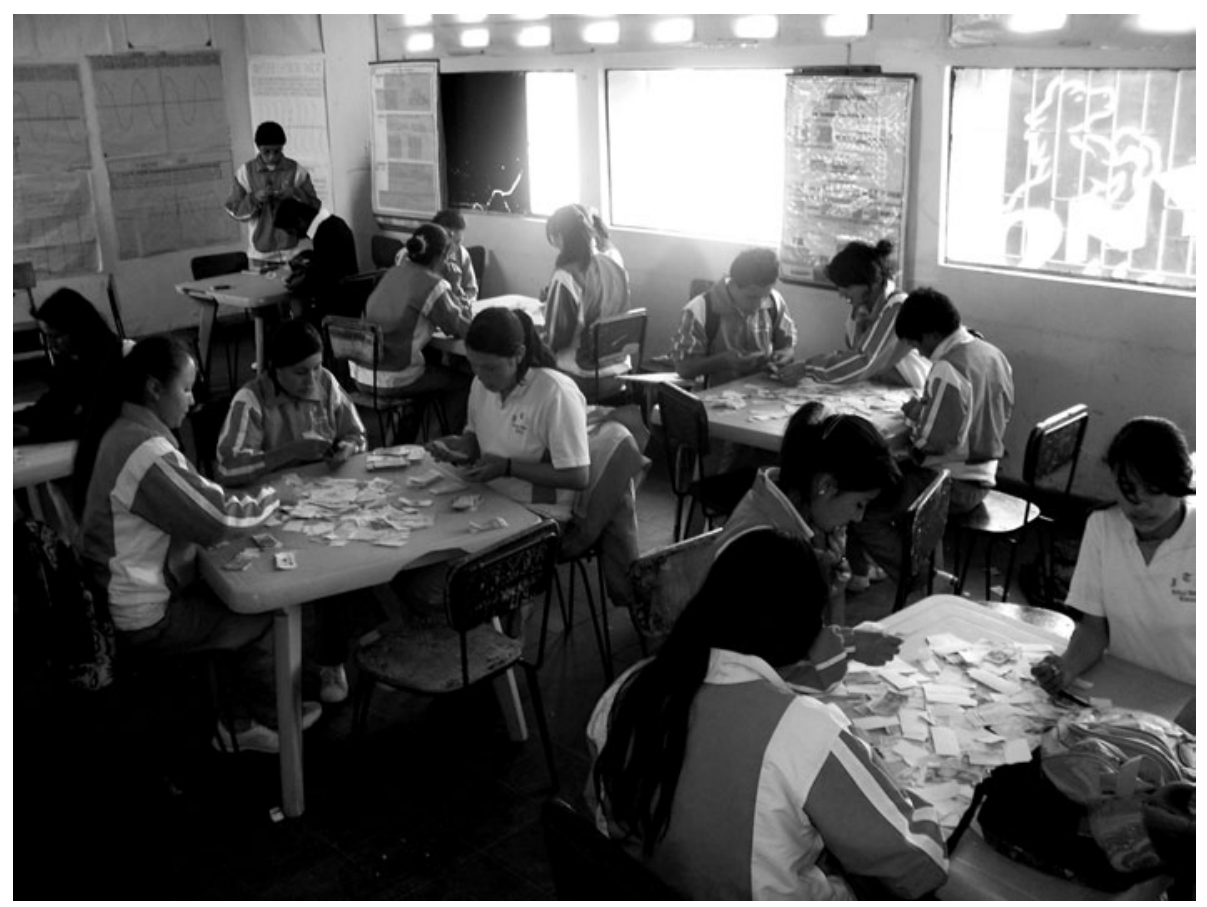

Imagen 1. Estudiantes de grado décimo en grupos de trabajo colaborativo, solucionando problemas sobre matemática financiera.

El ambiente de aprendizaje colaborativo favorece que los estudiantes de cada grupo avancen coordinadamente en el estudio de uno o más temas, por lo que los apuntes son semejantes y desde luego, la solución de las situaciones problema, lo que facilita 
al docente el proceso de evaluación para el grupo, ya que revisando un cuaderno de apuntes o trabajo grupal se están orientando a tres o cuatro estudiantes a la vez.

El proceso de evaluación en el entorno para el aprendizaje de las matemáticas se compone de cuatro etapas:

Una primera se realiza de forma continua sobre los avances que realiza el grupo, en la cual los estudiantes se apoyan mutuamente en la construcción del conocimiento. En esta etapa se encuentra implícita la evaluación diagnóstica, puesto que esta se realiza, no solo al inicio de un ciclo escolar, sino en diferentes momentos, como puede ser al principio de un tema o al comenzar una clase (López \& Hinojosa, 2001). En esta etapa, el docente hace las correcciones de los avances del grupo en forma escrita, en el cuaderno de un estudiante diferente cada vez o en el pliego que se entregue; las observaciones son detalladas sobre los procedimientos realizados y escribiendo frases como: "qué bien trabajaron el problema", "excelente su producción durante la clase", "las operaciones quedaron correctamente trabajadas, felicitaciones", "estudiando así llegarán lejos". Cuando los procedimientos de solución del problema presentan incoherencias, operaciones mal realizadas o resultados interpretados en forma equivocada, el docente sugiere por escrito, y en el sitio donde se cometió el error, las acciones necesarias para que los procedimientos hechos por los alumnos tengan sentido, nunca tacha (poner equis) o escribe la respuesta correcta, es tarea del estudiante, en actividades posteriores, encontrarla. En esta etapa de la evaluación no se asigna ningún número como valor por el trabajo hecho, pues previamente los estudiantes han sido sensibilizados sobre la importancia de aprender, por encima de un número que irá registrado sobre un papel y que en un futuro no representa mucho frente al desempeño en el campo profesional o la vida cotidiana fuera del aula. Además, el alumno saber que en la tercera etapa de la evaluación debe demostrar lo que en realidad es capaz de hacer con lo aprendido.

La segunda etapa de la evaluación consiste en presentar una actividad de forma individual, así: sobre un problema resuelto en clase de forma grupal (trabajo colaborativo), el docente formula preguntas de tipo inferencial, de tal manera que para encontrar la respuesta, el alumno pueda basarse en las actividades realizadas a lo largo del desarrollo del tema, en este caso, al estudiante se le permite consultar textos, apuntes, fichas, entre otros recursos. De esta manera, él puede hacer un proceso de reconstrucción y análisis, para llegar a conclusiones que le ayuden a afianzar su conocimiento, la retroalimentación se lleva a cabo de la misma forma que en la primera etapa.

En la tercera etapa, el estudiante tiene la oportunidad de formular y solucionar problemas relacionados con su entorno y que involucran el conocimiento construido durante determinado tiempo escolar (semestre o periodo académico). En esta 
etapa, él solo cuenta con lápiz y papel, es el momento en el cual pone a prueba el conocimiento, capacidad de redacción, pensamiento coherente, orden lógico en las ideas, uso de las unidades, estimación de cantidades, análisis, entre otras habilidades y competencias. La retroalimentación en esta actividad es mucho más detallada que la realizada en las anteriores, puesto que corresponde a la producción del estudiante, es lo que él sabe hacer con lo que aprende (MEN, 2009).

Por último, en la cuarta etapa el estudiante valora su aprendizaje, y lo expresa por medio de un número que esté acorde con las exigencias institucionales para ser promovido, pero... ¿y cómo aparecen las notas, entonces?; al respecto, Guillermo Torres Zambrano, en su artículo Aspectos a tener en cuenta en la planeación y desarrollo de acciones evaluativas (1996), dice que:

Es un deber del profesor generar un diálogo sincero, abierto y constructivo sobre la experiencia evaluativa. Y es deber del profesor y de los estudiantes asumir las consecuencias que deriven de este diálogo: aceptar la excelente o deficiente calidad de la experiencia, estar en posibilidad de admitir la experiencia tal como sucedió o rechazarla por la manera como sucedió, incluso, si se amerita como resultado de la discusión, plantear la posibilidad de hacer nulo el resultado de la evaluación. Todo lo anterior implica que la evaluación, los exámenes, como todos los eventos de enseñanza y aprendizaje, tienen un carácter relativo, opuesto al sentido absoluto que se le ha querido atribuir.

Este diálogo entre los actores (profesor y estudiantes, estudiantes entre sí) debe convertirse en oportunidad para cualificar la acción educativa, además de constituirse en un evento de participación que es muy importante en términos de la formación de personas, futuros profesionales y ciudadanos.

Luego esta actividad será la que ayude al docente y al estudiante, de común acuerdo, a través de una revisión cuidadosa y consciente sobre lo que fue el proceso de enseñanza y aprendizaje, a definir la nota que irá al sistema de calificaciones; en ningún caso el docente asigna números que califiquen el aprendizaje del estudiante, es este quien sabe realmente cuánto aprendió de lo propuesto, de lo que es capaz de hacer con lo aprendido, por ende, con mayor seguridad puede asignar la nota que se merece. El papel del profesor en esta etapa es el de persuadir al estudiante, a lo largo del diálogo (no más de cinco minutos) y con las evidencias del proceso (cuaderno, actividades grupales e individuales y producción final), para que su decisión final sea lo más justa posible.

\section{Resultados encontrados}

Al relacionar y poner en práctica los cuatro sistemas didáctico-pedagógicos mencionados en la propuesta denominada entorno para el aprendizaje de las 
matemáticas, antes planteada, se han observado manifestaciones emocionales, actitudinales y socioacadémicas en el estudiante frente a la construcción del conocimiento matemático.

\section{Expresiones emocionales del estudiante}

El paso de los estudiantes por el entorno para el aprendizaje de las matemáticas ha dejado varias impresiones, que invitan a la reflexión sobre las condiciones que hacen posible la educación del ser humano. Describiré algunas de las que han impactado a lo largo del desarrollo de la propuesta.

Desde el momento en que el estudiante ingresa al aula de matemáticas (entorno para el aprendizaje de las matemáticas), se percibe el cambio de expresión en su rostro; unos se impresionan por los trabajos que sus compañeros han dejado publicados en el salón; otros, por los mensajes que encuentran en la pantalla; otros, por la disposición del lugar para la clase, o sencillamente porque han llegado a un sitio diferente a los compartidos en forma cotidiana; las sonrisas, los chistes, las bromas no se dejan esperar, se convierten en uno de los ingredientes principales para mantener el dinamismo en la clase.

Al pedir la opinión de los estudiantes sobre la propuesta de tener un entorno para el aprendizaje de las matemáticas, escribieron frases como: "uno sale de la monotonía de un solo salón de clase", "se despeja la mente al cambiar de salón", "qué chévere es tener un salón solo para matemáticas", "nos sentimos libres", "nos sentimos más seguros y acompañados", "estudiamos con más gusto y menos tensionados, porque en la solución de los ejercicios, si uno no sabe o no entiende, los demás nos ayudan y nos rinde hacer los trabajos"; expresiones como estas dan a entender que el estudiante se siente mucho mejor construyendo conocimiento matemático en un entorno pensado y adecuado exclusivamente para ese fin.

Por otra parte, la implementación del sistema didáctico ABP en el desarrollo de las clases, ha permitido que el estudiante se sienta identificado con su entorno, esto lo induce a que proponga situaciones problema contextualizadas, lo cual genera en él seguridad al hablar, leer y escribir, y fortalece la confianza en sí mismo para elegir caminos que lo conduzcan a la solución de problemas propios, propuestos por el docente orientador de la asignatura o presentes en los textos de apoyo. 


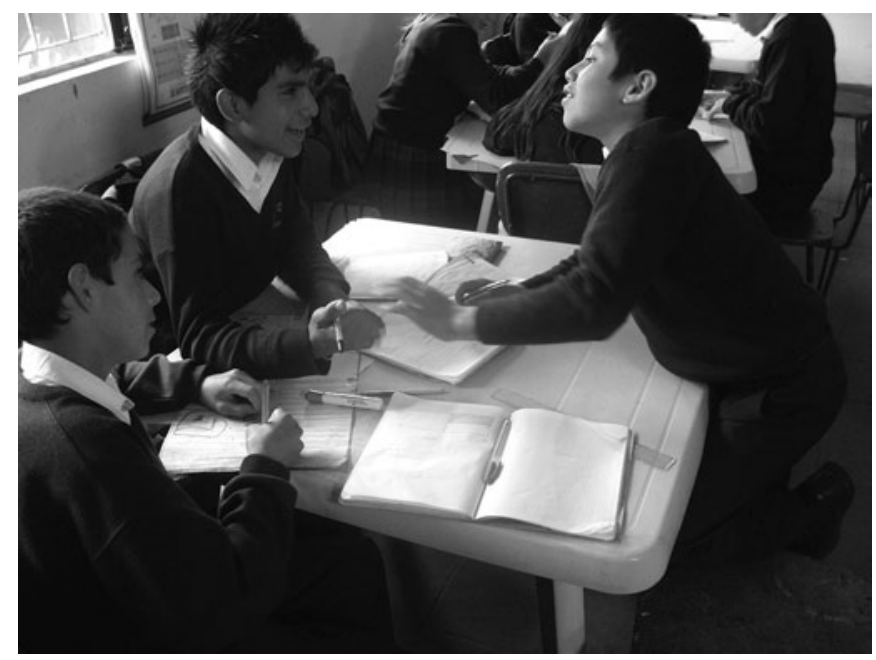

Imagen 2. Estudiantes de grado séptimo en clase de matemáticas, debatiendo ideas acerca de la resolución de un problema cotidiano sobre aplicación de las operaciones entre números naturales.

Un estudiante de grado séptimo redactó la siguiente situación problema, cuando se le pidió que aplicara las operaciones entre números naturales en situaciones cotidianas: "La señora Margarita llevó el sábado al pueblo 15 libras de queso para la venta; cada libra la vendió a \$3.300. Con parte del dinero de la venta del queso compró los siguientes artículos para la casa: \$ 1.000 de cebolla larga, \$2.000 de tomate, $\$ 3.200$ de banano, $\$ 1.500$ de zanahoria, $\$ 500$ de habichuela, $\$ 1.800$ de fríjol y un rollo de papel higiénico de $\$ 1.200$, el dinero que le sobró lo repartió entre ella y su esposo, en partes iguales". El estudiante formuló las siguientes preguntas: ¿cuánto dinero recibió doña Margarita por la venta del queso?, ¿cuánto dinero le correspondió a su esposo?

De la misma forma, la evaluación formativa ha despertado en el estudiante la necesidad de estudiar, no por la nota, sino para dar razón a su conciencia en el momento en que efectúa el análisis de su rendimiento académico (cuarta etapa de la evaluación de la presente propuesta), con sus respectivas evidencias y en presencia del docente. Es de notar que progresivamente el alumno acepta la evaluación formativa y se desprende de la costumbre de ver un número que califica sus saberes.

\section{Actitud del estudiante en el aula}

Al cambiar el ambiente de clase, el estudiante se relaja física y mentalmente, toma aire fresco, que lo dispone (despierta) para el desarrollo de las actividades propias de la asignatura. Al ingresar al entorno para el aprendizaje de las matemáticas, él manifiesta alegría y curiosidad por lo que puede encontrar allí, sonríe, como 
expresando un nuevo punto de partida en su quehacer académico, y observa con sumo cuidado los elementos que se encuentran en el lugar. Lo sorprendente es que los estudiantes que circulan por el recinto quieren estar en ese lugar, lo manifiestan directamente con expresiones como “¡oh!, qué chévere el salón”, otros preguntan: ¿ya se terminó la clase?, cuando suena el timbre para el cambio de asignatura y, por supuesto, de lugar, entre otras expresiones que no se pueden describir, solo se comprenden al estar con ellos. El aula representa para el estudiante la diferencia entre la monotonía de lo tradicional y el dinamismo e interacción que reclama la educación actual.

En el desarrollo de las clases de matemáticas, el estudiante manifiesta seguridad al hablar y exponer sus ideas frente a su grupo de trabajo o clase, también expresa compromiso institucional en actividades del área, talleres, tareas y horarios de clase. Los Ilamados de atención por indisciplina en la clase son ocasionales, esto hace que las relaciones académicas y sociales entre docente y estudiantes sean mucho más enriquecedoras y formativas.

\section{Expresiones socioacadémicas del estudiante}

La comodidad de tener los textos de diferentes grados en el aula, la conexión a internet, el video beam, el diverso material didáctico y la rotación de estudiantes de diferentes grados en cada clase le han dado vida al aula. Si en un momento determinado un estudiante no se acuerda de un tema, procedimiento, fórmula o concepto, puede consultar textos o internet inmediatamente. Los diversos elementos didácticos representan una excelente ayuda para el docente en el momento de explicar aspectos inesperados de la clase, o cuando los estudiantes están realizando talleres dentro de la misma.

Otro aspecto relevante es la actitud del estudiante frente a los trabajos que elabora, expone y fija en lugares visibles del salón, como estos llevan el nombre de los autores, se ha notado que hay mayor auto-exigencia en cuanto a la calidad de los mismos, esto inspira a estudiantes de otros grupos a elaborar trabajos cada vez mejores. El ejercicio de fijar los trabajos hechos por ellos en el aula de clase, a manera de exposición, deja ver el aprecio que tienen por lo que hacen, y lo expresan directamente cuando tienden a "pelearse" los lugares más visibles y elegantes del salón para tal fin, se nota que desean que otras personas los aprecien; por lo contrario, cuando los trabajos no quedan muy agradables, piden que se les permita mejorarlos en casa o en el recreo, para luego publicarlos. 


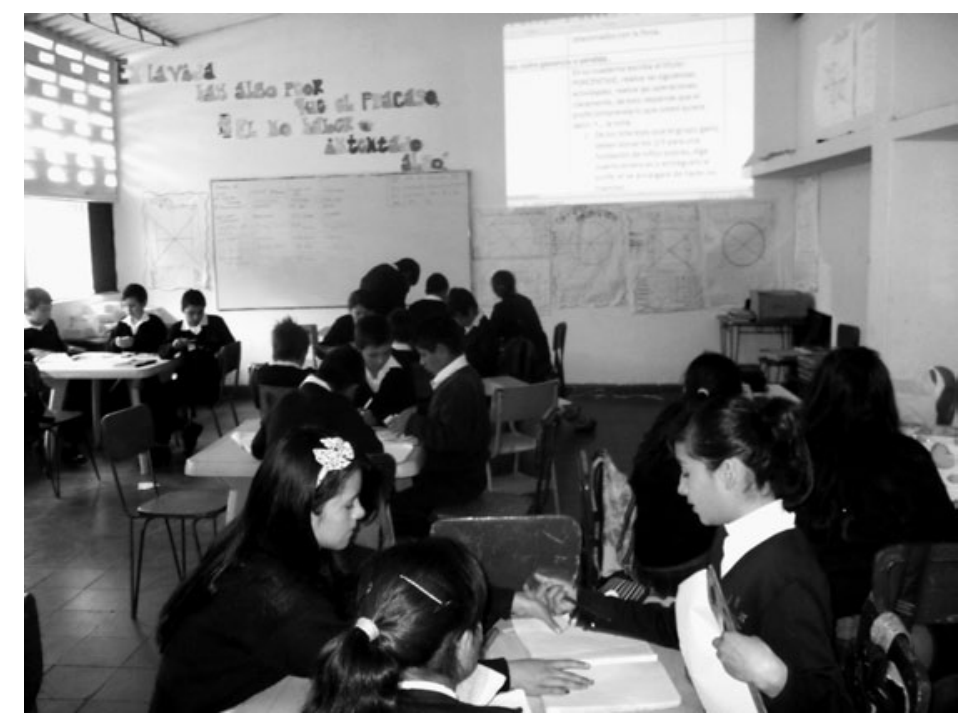

Imagen 3. Estudiantes en clase desarrollada en el entorno para el aprendizaje de las matemáticas, al fondo se ven expuestos algunos trabajos de estudiantes, el tablero donde ellos efectúan ejercicios de refuerzo y la pantalla del video beam con la estructura de la clase del día.

El ejercicio de publicar los trabajos en el salón fortalece valores y habilidades, desarrolla competencias, y hace posible descubrir aptitudes y talentos, también funcionan como material didáctico, sobre el cual se pueden orientar diversos conocimientos. Se ha podido percibir el orgullo (sano, por supuesto) y alegría que manifiestan los estudiantes cuando el docente se apoya en sus trabajos para explicar a otros grupos temas en desarrollo o para recordar conocimientos olvidados.

Una de las diferencias entre el desempeño del estudiante en el entorno para el aprendizaje de las matemáticas y el que tiene en un aula común, es que en el primero él manifiesta más seguridad al hablar del tema de la clase con sus compañeros o cuando el docente entabla una conversación con el grupo, y formula preguntas de control sobre el tema. En el salón común manifiesta inseguridad, quizá miedo a hacer el ridículo frente a sus compañeros y profesor, o simplemente responde "no sé".

Cuando los problemas de los cuales se parte para la enseñanza de determinado tema pertenecen al contexto del estudiante, se ha notado que le es más fácil comprender, se percibe que propone con mayor facilidad posibles soluciones, actúa con más seguridad. En el análisis que efectúa usa términos que para él son conocidos, lo que le permite una mejor comunicación con los demás integrantes del grupo. La clase se convierte en un ambiente familiar en el que se construyen conocimientos matemáticos útiles para el estudiante en su quehacer cotidiano, a 
la vez, se desmitifica la idea de que las matemáticas son sólo para personas con habilidades excepcionales, o que son muy difíciles de aprender y que nunca se usa lo que se aprende en el aula.

La responsabilidad frente al estudio es otro aspecto en el que los estudiantes han manifestado cambios, quienes no tomaban apuntes, o lo hacían de forma desordenada, han venido mejorando progresivamente, pues ellos saben que en cualquier momento su cuaderno representará al grupo, será evaluado, y es la imagen del mismo la que está en juego. Por otra parte, los alumnos a los que les gustaba aprobar la asignatura con las notas que obtenían por los trabajos grupales, que promediadas con las malas notas de evaluaciones individuales se daban el lujo de pasar sin estudiar y, peor aún, sin aprender, saben que si no despejan las dudas en el trabajo grupal, cuando se enfrenten a su propia realidad, a través de la evaluación formativa en cada una de sus etapas, no podrán justificar una nota aprobatoria para la asignatura, y lo peor para ellos es que el grupo los rechace, los aíslen sus propios amigos, y eso no es aspecto que les agrade mucho, luego no les queda otra opción que aportar al trabajo, como todos lo hacen.

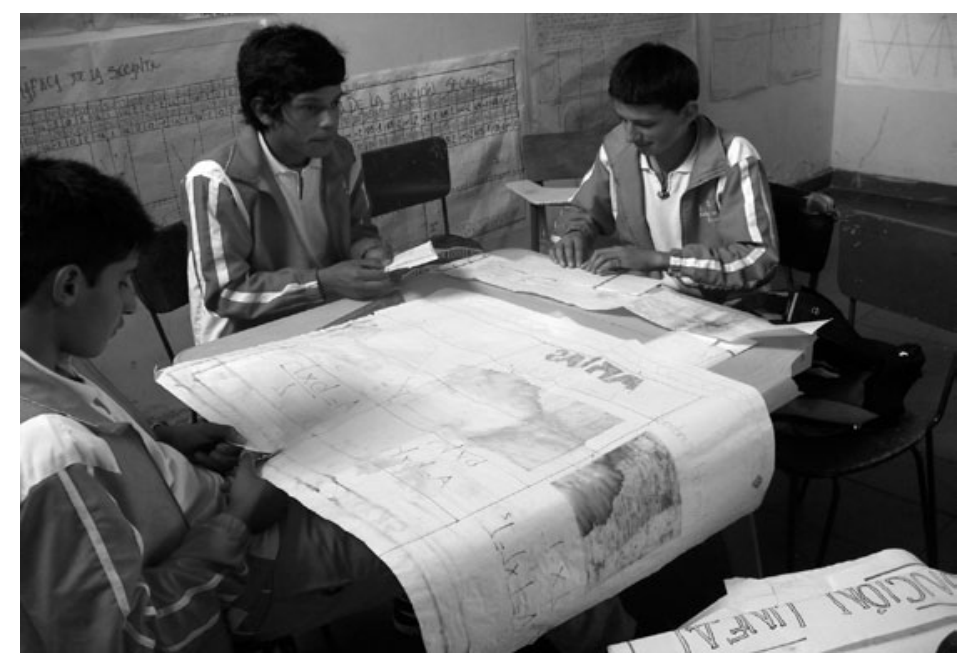

Imagen 4. Estudiantes de grado noveno diseñando material didáctico sobre área de figuras planas, para ser expuesto en el aula de clase como apoyo al aprendizaje en niños de otros grados.

Además, se ha notado una mejor comprensión y aprendizaje de los temas de la asignatura, la clase es mucho más relajada, los Ilamados de atención por rendimiento académico han disminuido, la comunicación entre docente-estudiante y estudianteestudiante es mucho más fluida y de confianza. Al estar el docente asesorando a los grupos continuamente le permite conocer mucho más a cada estudiante, este pasa de ser el número tal de la lista o el que se sienta en la fila equis, a ser el que 
se expresa de tal forma, que tiene tales ideas, sus amigos son tales, su letra es de tal forma, etc. El docente guarda la imagen del alumno por sus aportes al grupo y en la clase, mas no por la ubicación en el salón de clases o por los números que se registran en una planilla.

Del trabajo colaborativo se resaltan las sanas discusiones entre los estudiantes sobre aspectos de la clase, cuando no se ponen de acuerdo consultan a otros grupos, o en textos, o piden ayuda al profesor para estar seguros sobre la posible decisión que tomen, puesto que algunos problemas requieren de análisis detallados y de procedimientos específicos para su solución. Cuando el grupo se enfrenta a un nuevo problema manifiesta inseguridad, timidez, pero siempre hay uno o varios que son aventajados en matemática, y tratan de llevar al vocabulario común la terminología correspondiente, así el grupo lentamente, va encontrando la ruta de trabajo. En muchas ocasiones prescinden de la ayuda del docente, lo que indica que están ganando confianza en sí mismos, responsabilidad e independencia en la construcción del conocimiento.

En este proceso se construyen más que conocimientos matemáticos, se fortalecen valores como el respeto, la tolerancia, los buenos modales, la alteridad, el buen trato, entre otros. Se corrigen entre sí la forma de expresarse y actuar, unifican criterios frente a vivencias cotidianas, establecen puntos de referencia, en otras palabras, hay construcción social de conocimiento y práctica de valores.

\section{Conclusiones}

El estudiante de hoy necesita entornos de aprendizaje diferentes a los tradicionales, que lo motiven contantemente a la construcción del conocimiento matemático, que estén acordes con los cambios tecnológicos y sociales, tendientes a fortalecer en él habilidades y competencias útiles en su desempeño cotidiano, que lo potencien para enfrentar un mundo cada vez más exigente, cambiante y globalizado.

Un entorno para el aprendizaje de las matemáticas, donde se conjugan aula especializada, ambiente de estudio colaborativo, aprendizaje basado en problemas y un sistema de evaluación formativa, proporciona al estudiante una amplia gama de posibilidades para que él construya socialmente el conocimiento matemático de manera reflexiva sobre su propio quehacer cotidiano.

Cuando se pidió a un grupo de docentes conocedores del desarrollo de la propuesta que opinaran sobre la misma, sus comentarios fueron bastante espontáneos: "es interesante la metodología, sobre todo por el trabajo colaborativo"; "es una mirada diferente sobre los aspectos metodológicos"; "es una propuesta muy acertada, puesto que le da un papel protagónico al estudiante"; "el ambiente dispone al 
estudiante para que construya el conocimiento"; "permite al estudiante comprender con mayor facilidad los temas"; "permite romper la monotonía de lo tradicional"; "esta metodología motiva al estudiante"; "se torna valioso el trabajo colaborativo, se fortalecen valores, se saca al estudiante de la rutina"; "es una propuesta interesante y enriquecedora"; "rompe esquemas tradicionales de enseñanza y aprendizaje"; "existe más identidad del docente y los estudiantes con el conocimiento". De igual manera, manifestaron con bastante convicción que la propuesta se puede extender a todas las áreas del conocimiento que se oriente en la institución. 


\section{Referencias}

Barrows, H y Tamblyn, R. (1980). Problem-based learning: An approach to medical education. New York: Springer.

Bruffee, K. (1995). Sharing our toys: Cooperative learning versus collaborative learning. Change, 27 (1), 12-18.

Collazos, C. y Mendoza, J. (2006). Cómo aprovechar el "aprendizaje colaborativo" en el aula. Educación y Educadores, 9 (2), 61-78.

D’Amore, B. Godino, J y Fandiño, M. (2008). Competencias y matemáticas. Bogotá: Cooperativa Editorial Magisterio.

Dillenbourg, P. (1999). Collaborative Learning: Cognitive and Computational Approaches. Oxford: Pergamon Press.

Escribano, A., Del Valle, Á. (2010). El aprendizaje basado en problemas. Madrid: Narcea.

Fandiño, M. (2006). Currículo, evaluación y formación docente en matemáticas. Bogotá: Cooperativa Editorial Magisterio.

Fernández, P., Melero, M.A. (1995). La interacción social en contextos educativos. Madrid: Siglo XXI.

Gardner, H. (1995). La evaluación en su contexto. La alternativa a los test estandarizados. En H. Gardner. Inteligencias múltiples. La teoría en la práctica. Barcelona: Paidós.

Hmelo-Silver, C.E. (2004). Problem-based learning: What and how do students learn? Educational Psychology Review, 16, 235-266.

Johnson, D.W., Johnson, R., y Holubec, E. (1993). Circles of learning (4th ed.). Edina, MN: Interaction Book Company.

López, B. Hinojosa E. (2001). Evaluación del aprendizaje. Alternativas y nuevos desarrollos. México: Trillas.

Mejía, M. (2011). La sistematización. Empodera y produce saber y conocimiento. Bogotá: desde abajo.

MEN (1994). Ley General de Educación 115 de 1994. Bogotá.

MEN (2009). Estándares de matemáticas. Bogotá.

Puentes, Y. (2005). Organizaciones escolares inteligentes, gestión de entornos educativos de calidad. Bogotá: Cooperativa Editorial Magisterio.

Santaibáñez, J. (2001). Manual para la evaluación del aprendizaje estudiantil. México:Trillas S.A.

Santos, M. (1995). La evaluación: un proceso de diálogo, comprensión y mejora. Granada: Ediciones Aljibe S.L. 
Torres, G. (1996). Aspectos a tener en cuenta en la planeación y desarrollo de acciones evaluativas. Bogotá.

Recibido: 30 septiembre 2013

Aceptado: 28 noviembre 2013

Cómo citar:

López-Quijano, G. (2014). La enseñanza de las matemáticas, un reto para los maestros del siglo XXI. Praxis Pedagógica, 15, 55-76 